\title{
The Chemistry of Kitols
}

\author{
Kiyoshi TsukIDA* and Masayoshi ITo* \\ (Received July 23, 1980)
}

\begin{abstract}
The structure of kitol, a natural retinol dimer, was reinvestigated. Kitol-A and -B were isolated from whale liver and the former was recognized as "kitol" reported so far. Extensive ${ }^{1} \mathrm{H}-\mathrm{NMR}$ $(200 \mathrm{MHz}$ ) data for kitol-A and its related compounds (retinyl acetate-tetracyanoethylene adduct, -fumaronitrile adduct, and -crotononitrile adduct) were presented and the structure of kitol-A including its stereochemistry was determined decisively. Kitol-B is believed to be the mono-cis isomer of kitol-A.
\end{abstract}

Since the discovery of kitol, a natural retinol dimer initially found in whale liver oil, ${ }^{12}$ a large number of papers on its chemical structure have been published, ${ }^{2)}$ i.e. structures $(\mathbf{I}),{ }^{8,4)}(\mathbf{I I})^{5)}$ and $(\text { III) })^{8,7)}$ were successively proposed for this natural product, while (IV) ${ }^{4)}$ for a pyrolysis product pseudokitol (Fig. 1). Kitol has since been regarded as a 1,4-cycloaddition product of the 11 , 13-diene part of the first with the 13-monoene part of the second retinol molecule, leading to the formation of the central cyclohexene ring. Until recently, based mainly on $60 \mathrm{MHz}^{1} \mathrm{H}-\mathrm{NMR}$ data ${ }^{3}$ or on mass spectral evidence, ${ }^{7)}$ the structure of the parallel addition type III has been generally accepted as the most favorable chemical structure of kitol, while the photodimerization of retinyl esters $^{8-11)}$ and chemical formation of kitol from retinol ${ }^{12,13)}$ have also been investigated in detail. In 1976, however, YANO $^{14)}$ proposed a novel cyclopentane structure $(\mathbf{V})$ and claimed an invalidity of the formula III described above. This statement prompted us to report our findings on the chemical structure and stereochemistry of kitol. ${ }^{16)} \mathrm{We}$ now give here a full account of our results on the chemistry of kitol-A and -B together with their related compounds such as the tetracyanoethylene adduct (VI), ${ }^{18)}$ the fumaronitrile adduct (VII) and the crotononitrile adduct (VIII) with retinyl acetate (Fig. 1).

Crude kitol, isolated from whale liver, was successfully divided into two components, designated kitol-A (Rf 0.51) and kitol-B (Rf 0.45), by employing preparative thin-layer chromatography (pTLC) on a silica-gel plate. Although both kitol-A and -B have similar spectral characteristics, all experimental results demonstrated that the hitherto known kitol was in excellent agreement with kitolA but not with kitol-B.

It is obvious that the most important information for distinguishing the structure III from $\mathrm{V}$ can be obtained directly from ${ }^{1} \mathrm{H}$ - or ${ }^{13} \mathrm{C}-\mathrm{NMR}$ data, e.g. the existence of the particular proton or carbon such as $11-\mathrm{H}$ or 11-C in $\mathrm{III}$ and $14-\mathrm{H}$ in $\mathrm{V}$, number of protons attached to the carbinol carbons, or splitting pattern(s) of carbinol carbons in offresonance measurement would be the point for this purpose. A number of NMR measurements employing various techniques were therefore undertaken. Of these, ${ }^{1} \mathrm{H}-\mathrm{NMR}$ of kitol-A was completely resolved for the first time by the present authors, though assignment of some ${ }^{13} \mathrm{C}-\mathrm{NMR}$ signals remained uninterpreted. Careful inspection of the $200 \mathrm{MHz}{ }^{1} \mathrm{H}-\mathrm{NMR}$ data (Tables 1 and 2; Fig. 2) has eventually led us to the first recognition of the existence of the proton attached to a double allylic carbon atom, viz. 11-H in VI, VI, VIII, and then in III. Thus, it is now evident that the formula III is correct and V should be abandoned.

For determining the stereochemistry of kitol, BURGER et al. ${ }^{2)}$ attributed two one-proton-doublets at $\delta 5.42(\mathrm{~J} 6 \mathrm{~Hz})$ and $5.14 \mathrm{ppm}(\mathrm{J} 10.5 \mathrm{~Hz})$ to $10-\mathrm{H}$ and $12-\mathrm{H}$, respectively ( $c f$. Table 2 ). Based on this interpretation they suggested that the trienyl chain attached to the 11-C position of the central ring would be in the quasi-axial conformation. However, from extensive decoupling experiments on kitol-A and its related compounds, especially on VII, we conclude that Burger's assignment on $10-\mathrm{H}$ and $12-\mathrm{H}$ as well as stereochemical interpretation around 11-C should now be revised as follows. We ascribe the one-proton-doublet at

* Kobe Women's College of Pharmacy, Motoyama-kitamachi, Higashinada-ku, Kobe 658 （月田 潔 - 伊 藤允好：神户女子薬科大学). 
<smiles>[R]C=CC1(C)C(CO)C(CO)C1(C)/C=C/[R]</smiles>

( I)<smiles>[R]C=CC1(C)CC(O)C(C)(/C=C/[R])CC1O</smiles>

(II)<smiles>[R]C=CC1(/C=C\CO)C[C@H](O)C(C)/C1=C/CO</smiles>

(V)

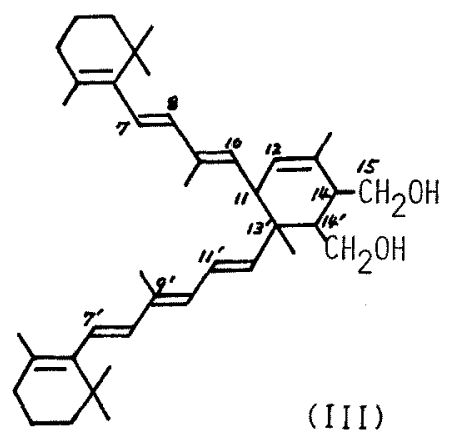

(IV)<smiles>[R]C=CC1(C)C(CO)C(C)=CC([R])C1C</smiles>

(IV)

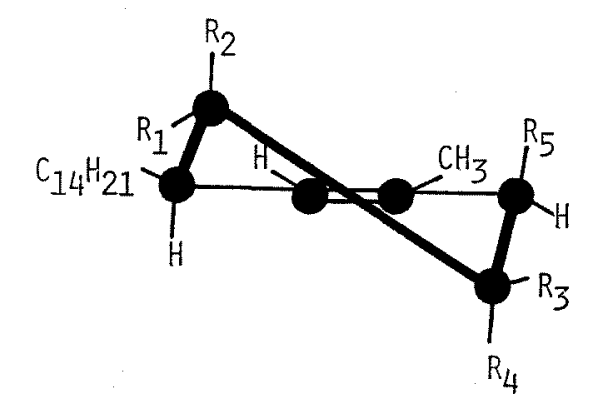

(IIIa): $\mathrm{R}_{1}=\mathrm{C}_{16} \mathrm{H}_{23}, \mathrm{R}_{2}=\mathrm{CH}_{3}$ $\mathrm{R}_{3}=\mathrm{R}_{5}=\mathrm{CH}_{2} \mathrm{OH}, \mathrm{R}_{4}=\mathrm{H}$

(VIIa): $R_{1}=R_{3}=C N, R_{2}=R_{4}=H$ $\mathrm{R}_{5}=\mathrm{CH}_{2} \mathrm{OAC}$

Fig. 1. The structures of kitol and model compounds.

$\delta 5.15 \mathrm{ppm}$ to $10-\mathrm{H}$ and the broad one-protonsinglet centered at $5.36 \mathrm{ppm}$ (see Footnote $* 4$ in Table 2) to $12-\mathrm{H}$. Moreover, $J_{10,11}$ and $J_{11,12}$ values are newly estimated as 9 and $c a .0 \mathrm{~Hz}$, respectively, instead of 6 and $10.5 \mathrm{~Hz}$ as reported earlier. It would therefore be apparent that the above mentioned trienyl side chain orients in the quasi-equatorial direction at 11-C on a twist halfchair form of the cyclohexene ring. It was confirmed that kitol-A has similar NMR spectral characteristics as model compounds (VI-VIII), amongst which the stereochemistry of the adduct VII was determined decisively as (VIIa). Consequently, with acceptance of the validity of the stereochemical conclusion for the adducts and based not only on the spectral but also on the chemical information accumulated so $\mathrm{far}^{2}{ }^{2}$ the configuration (IIIa) (trienyl and tetraenyl chains are trans [equatorial and quasi-equatorial] and two hydroxymethyl groups are cis [equatorial and quasi-axial]) would be expected for kitol-A. This deduction involves further argument ${ }^{11}$ that the orbital overlap would proceed dominantly at 14 $14^{\prime}$ rather than at $12-12^{\prime}$ in a concerted cycloaddition reaction of the first to the second retinol molecule.

Finally, kitol-B, the second component of a crude kitol isolated from whale liver, was investigated. At the present, it seems reasonable to determine kitol-B as the $9^{\prime}$-monocis isomer of kitol-A (all-trans form) from the following spectral features: $\lambda_{\max }$ in ethanol, 286 and 230 (infl.) $\mathrm{nm}$; 


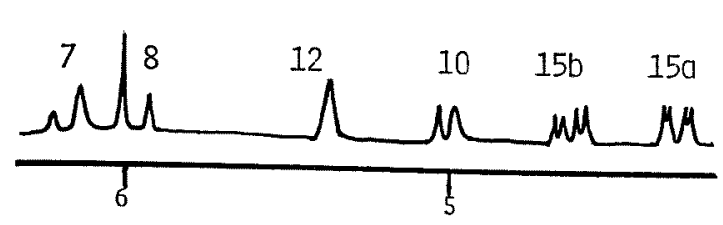

Adduct VII
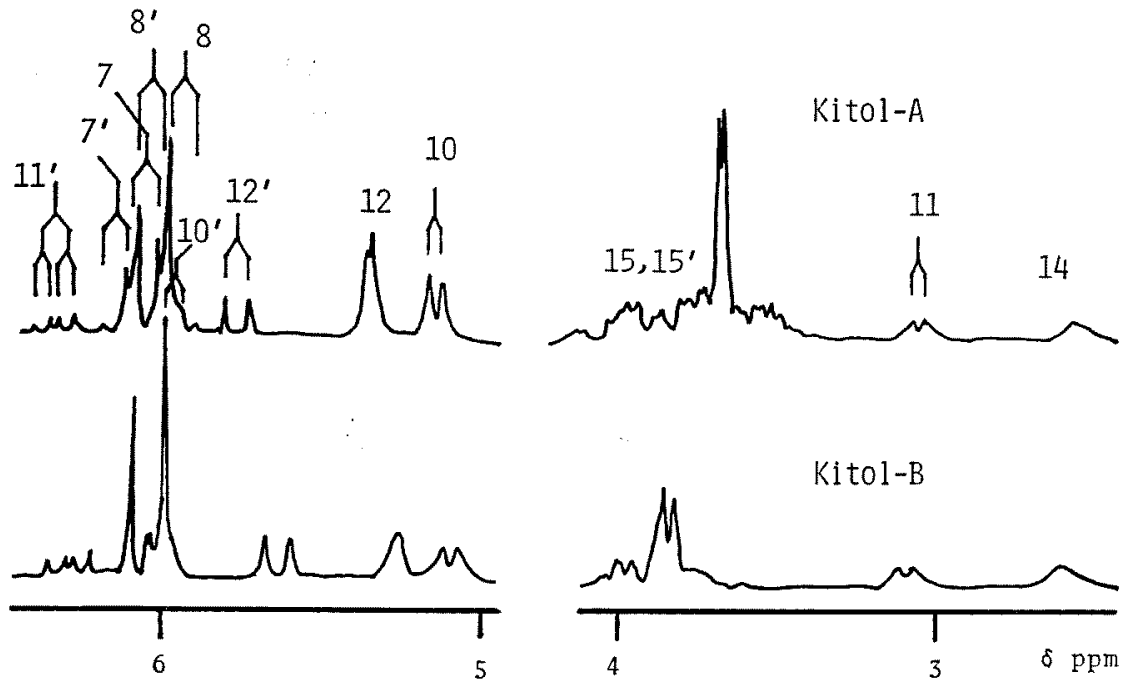

Fig. 2. ${ }^{1} \mathrm{H}-\mathrm{NMR}$ in $\mathrm{CDCl}_{3}$ for kitol (III) and the retinyl acetate-fumaronitrile adduct (VII). (200 $\mathrm{MHz} ; \delta$ ppm from internal tetramethylsilane; resonances in a higher magnetic field were omitted).

Table 1. ${ }^{1} \mathrm{H}-\mathrm{NMR}$ chemical shifts in $\mathrm{CDCl}_{3}(\delta \mathrm{ppm}$ from internal tetramethylsilane) for the model compounds (VI)-(VIII)

\begin{tabular}{|c|c|c|c|c|c|c|c|c|c|}
\hline \multirow{2}{*}{ Compound } & \multirow{2}{*}{$\mathrm{MHz}$} & \multicolumn{6}{|c|}{$\mathrm{CH}_{3}$} & \multicolumn{2}{|c|}{$\mathrm{CH}_{2}$} \\
\hline & & 1,1 & 5 & 9 & 13 & OAc & $b^{\prime}$ & $15 \mathrm{a}$ & $15 b$ \\
\hline VI & 90 & $\begin{array}{l}1.01 \\
(s)\end{array}$ & ${ }_{(s)}^{1.70}$ & $\begin{array}{l}2,02 \\
(d, 1)^{* 1}\end{array}$ & $\begin{array}{l}1.95 \\
\text { (br.s) }\end{array}$ & $\begin{array}{l}2.18 \\
(s)\end{array}$ & - & $\begin{array}{l}4.46 \\
(d d)\end{array}$ & $\begin{array}{l}4.82 \\
(d d)\end{array}$ \\
\hline VII & 200 & $\begin{array}{l}1.01 \\
(s)\end{array}$ & $\begin{array}{l}1.69 \\
(s)\end{array}$ & $\begin{array}{l}1.94 \\
(d, 1.3)\end{array}$ & $\begin{array}{l}1.79 \\
\text { (br.s) }\end{array}$ & $\begin{array}{l}2.12 \\
(s)\end{array}$ & - & $\begin{array}{l}4.29 \\
(d d)\end{array}$ & $\begin{array}{l}4.63 \\
(d d)\end{array}$ \\
\hline VIII & 200 & $\begin{array}{l}1.01 \\
(s)\end{array}$ & $\begin{array}{l}1.68 \\
(d, 0.4)\end{array}$ & $\begin{array}{l}1.93 \\
(d, 0.7)\end{array}$ & $\begin{array}{l}1.76 \\
(t, 0.8)\end{array}$ & $\begin{array}{l}2.06 \\
(s)\end{array}$ & $\begin{array}{l}1,28 \\
(d, 6.8)\end{array}$ & $\begin{array}{l}4.05 \\
(d d)\end{array}$ & $\begin{array}{l}4.39 \\
(d d) \\
\end{array}$ \\
\hline \multirow{2}{*}{ Compound } & \multirow{2}{*}{$\mathrm{MHz}$} & \multicolumn{4}{|c|}{$\mathrm{CH}$} & \multicolumn{4}{|c|}{ Olefinic $\mathrm{H}$} \\
\hline & & 11 & 14 & $a^{\prime}$ & $\mathrm{b}^{\prime}$ & 7 & 8 & 10 & 12 \\
\hline VI & 90 & $\begin{array}{c}4.14 \\
(m \text { of } d)\end{array}$ & $\begin{array}{l}3.34^{* 2} \\
(\mathrm{~m})\end{array}$ & - & - & $\begin{array}{l}6.38 \\
(d)\end{array}$ & $\begin{array}{l}6.07 \\
(d)\end{array}$ & $\begin{array}{l}5.46 \\
\text { (d) }\end{array}$ & $\begin{array}{l}5.62 \\
\text { (br.s) }\end{array}$ \\
\hline VII & 200 & $\begin{array}{l}3.55 \\
(t ?)\end{array}$ & $\begin{array}{l}2.65^{* 2} \\
\text { (br.s) }\end{array}$ & $\begin{array}{l}3.11 \\
(d d)\end{array}$ & $\begin{array}{l}3.31 \\
(d d)\end{array}$ & $\begin{array}{l}6,18 \\
(d)\end{array}$ & $\begin{array}{l}5.97 \\
(d)\end{array}$ & $\begin{array}{l}5.01 * 2 \\
(d)\end{array}$ & $\begin{array}{l}5.38 \\
\text { (br.s) }\end{array}$ \\
\hline VIII & 200 & $\begin{array}{l}3.46^{* 2} \\
(t ?)\end{array}$ & $\begin{array}{r}\simeq 2.5 \\
(\mathrm{~m})\end{array}$ & $\begin{array}{l}2.55 \\
(t ?) \\
\end{array}$ & & $\begin{array}{l}6.11 \\
(d)\end{array}$ & $\begin{array}{l}5.95 \\
(d)\end{array}$ & $\begin{array}{l}5.01 * 2 \\
(d) \\
\end{array}$ & $\begin{array}{l}5.29 \\
\text { (br.s) }\end{array}$ \\
\hline $\mathrm{J}(\mathrm{Hz})$ & $7-8$ & $10-11$ & $11-$ & \multicolumn{2}{|c|}{$\begin{array}{l}14-15 a \\
14-15 b\end{array}$} & $15 a-15 b$ & $11-a^{\prime}$ & $a^{\prime}-b^{\prime}$ & $14-b^{\prime}$ \\
\hline VI & 16 & 10 & 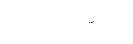 & 4 & 8 & 12 & - & - & - \\
\hline VII & 16.2 & 10.0 & & & 4.6 & 12.4 & 9.7 & 11.7 & 5.4 \\
\hline VIII & 15.8 & 9.9 & & & 4.6 & 12.0 & $\simeq 11$ & $\simeq 11$ & \\
\hline
\end{tabular}

-1 Splitting pattern and $\mathrm{J}$ value in $\mathrm{Hz}, \quad s$ singlet, $d$ doublet, $d d$ doublet of doublets, $t$ triplet, $t$ ? triplet-like, $m$ multiplet, br broad.

*2 Irradiated proton in decoupling experiments. 
44.19 (13'-C?), 46.35, 47.64, 63.94, 65.47, 124.93 $\left(10^{\prime}-\mathrm{C}+10-\mathrm{C} ?+12-\mathrm{C} ?\right), \quad 126.20\left(7,7^{\prime}-\mathrm{C}\right), \quad 127.60$ $\left(5,5^{\prime}-\mathrm{C}\right), 130.19\left(10^{\prime}-\mathrm{C}\right), 130.86\left(12^{\prime}-\mathrm{C} ?\right), 131.64$ (13-C?), $137.71\left(9,9^{\prime}-\mathrm{C}\right)$ and $137.85\left(6,6^{\prime}, 8,8^{\prime}-\mathrm{C}\right)$.

Diacetate, MS (sample temp, $200^{\circ} \mathrm{C}$ ) $\mathrm{M}^{+} \mathrm{m} / \mathrm{e}$ 656.479 (caled. for $\mathrm{C}_{44} \mathrm{H}_{64} \mathrm{O}_{4}, 656.481$ ), m/e 596 (M-HOAc), 536 (M-2HOAc), 480, 422, 407, $404,368,344,343,328\left(\mathrm{M}^{+} / 2\right.$, base), 268 (328HOAc), 255 (328- $\left.\mathrm{CH}_{2} \mathrm{OAc}\right), 253$ (328-HOAc-Me) and 199.

\section{Kitol-B $\left(9^{\prime}\right.$-monocis-III)}

UV 286 and 230 (infl.) nm; MS (sample temp. $\left.200^{\circ} \mathrm{C}\right) \mathrm{M}^{+}$?, m/e 368, $286\left(\mathrm{M}^{+} / 2\right.$ ?, base $), 271$, 268, 255, 223 and $199 ;{ }^{1} \mathrm{H}-\mathrm{NMR}$ see Table $2 ;{ }^{13} \mathrm{C}$ NMR $\delta$ ppm 12.98 (9-Me), 21.96 (9'-Me), 130.30 (8'-C) and $137.86(8-\mathrm{C})$. Diacetate, MS (sample temp. $200^{\circ} \mathrm{C}$ ) $\mathrm{M}^{+} \mathrm{m} / \mathrm{e} 656.484$ (calcd. for $\mathrm{C}_{44} \mathrm{H}_{64} \mathrm{O}_{4}$, $656.481)$.

\section{Retinyl Acetate-Tetracyanoethylene Adduct (VI)}

The adduct was prepared according to the previous report. ${ }^{18)} \mathrm{Mp} .229^{\circ} \mathrm{C},{ }^{1} \mathrm{H}-\mathrm{NMR}$ see Table 1.

\section{Retinyl Acetate-Fumaronitrile Adduct (VII)}

A mixture of fumaronitrile (trans-1,2-dicyanoethylene) $(0.75 \mathrm{~g})$ and retinyl acetate $(2.52 \mathrm{~g})$ in toluene $(30 \mathrm{ml})$ was heated in a sealed tube at $150^{\circ} \mathrm{C}$ (bath temp.) for $4 \mathrm{~h}$. After evaporated the solvent, the residue was purified through an alumina column (deactivated with $5 \% \mathrm{H}_{2} \mathrm{O}$ ) employing $3 \%$ acetone in benzene and was recrystallized from benzene-ether. Yield $0.66 \mathrm{~g}$. Rf (TLC, developed with ether-hexane 1:1) 0.38 ; mp. $154^{\circ} \mathrm{C}$; UV 265 ( $\varepsilon$ 22,000) and 235 ( $\left.\varepsilon 23,000\right)$ $\mathrm{nm}$; IR $\left(\mathrm{CHCl}_{3}\right) 2250(\mathrm{CN}), 1740+1230+1050$ (OAc) and 970 (trans- $\mathrm{CH}=\mathrm{CH}-$ ); MS (sample temp. $130^{\circ} \mathrm{C}$ ) $\mathrm{M}^{+} \mathrm{m} / \mathrm{e} 406.262(65 \%$ ) (calcd. for $\left.\mathrm{C}_{20} \mathrm{H}_{34} \mathrm{~N}_{2} \mathrm{O}_{2}, 406.262\right), 391$ (M-15, 13\%), 347 (MAcO), 346 (M-HOAc, 10\%), 333 (M-CH $\mathrm{CH}_{2} \mathrm{OAc}$ ), 331 (M-15-HOAc), 328 ( $\mathrm{M}-[\mathrm{NCCH}=\mathrm{CHCN}]$ ), 189 (M-side chain $\mathrm{C}_{14} \mathrm{H}_{21}, 23 \%$ ) and $119(100 \%)$; ${ }^{1} \mathrm{H}-\mathrm{NMR}$ see Table 1.

\section{Retinyl Acetate-Crotononitrile Adduct (VIII)}

A mixture of crotononitrile $(1.02 \mathrm{~g})$ and retinyl acetate $(0.5 \mathrm{~g})$ in xylene $(15 \mathrm{~m} l)$ was heated in a sealed tube at $170^{\circ} \mathrm{C}$ (bath temp.) for $7 \mathrm{~h}$. After evaporated the solvent, the residue was divided into two components on a pTLC plate. The upper band: UV 271 and $241 \mathrm{~nm}$; ${ }^{1} \mathrm{H}-\mathrm{NMR}$ see Table 1. The lower hand: UV 271 and 238 (infl.).

\section{Acknowledgment}

The authors gratefully acknowledge the expert technical assistance of Misses J. Kawabata, K. Tsuruta, T. Hirai and Y. Okabayashi. They also thank Dr. T. SHImIzU and Dr. K. YamaguchI for generous gifts of whale liver.

\section{References}

1) H. Pritchard, H. Wilkinson, J. R. Edisbury, and R. A. MORTON: Biochem. J., 31, 258-265 (1937).

2) B. V. Burger, and C. F. Garbers: J. Chem. Soc. Perkin I, 590-595 (1973), and papers cited therein.

3) R. Kaneko: J. Chem. Soc. Jpn., 80, 177-180 (1959).

4) Y. Oмоте: J. Chem. Soc. Jpn., 80, 191-197 (1959).

5) T. Yano and T. TomiYama: Bull. Japan. Soc. Sci. Fish., 29, 887--888 (1963).

6) B. V. Burger, C. F. Garbers, K. Pachler, R. Bonnett, and B. C. L. WeEdon: Chem. Commun., 588-589 (1965).

7) C. Giannotti, B. C. Das, and E. Lederer: Chem. Commun., 28-29 (1966); Bull. Soc. Chim. Fr., 3299-3303 (1966).

8) R. Kaneko: J. Chem. Soc. Jpn., 79, 1459-1460 (1958).

9) M. Mousseron-Canet, J.-C. MANi, and D. LERNER: Bull. Soc. Chim. Fr., 3043-3044 (1966).

10) C. Giannotti: Can. J. Chem., 46, 3025-3032 (1968).

11) M. Mousseron-Canet, D. Lerner, and J.-C. MANI: Bull. Soc. Chim. Fr., 4639-4645 (1968).

12) M. C. Ghosh, M. Rahman, and S. Ghosh: Indian J. Biochem. Biophys., 10, 289-290 (1973).

13) K. K. DAs: Ph. D. thesis, Gauhati University, 1970.

14) T. Yano: Sci. Bull. Fac. Agr., Kyushu Univ., 31, $41-47$ and $49-56$ (1976).

15) K. Tsukida and M. Ito: J. Nutr. Sci. Vitaminol, 26, 319-322 (1980).

16) K.-H. Pfoertner: Helv. Chim. Acta, 58, 833839 (1975).

17) T. TAWARA and R. Fukazawa: Bull. Japan. Soc. Sci. Fish., 16, 375-376 (1951). 